\title{
Statistical analysis of the X-ray flares $(M \geq 1)$ during the maximum period of solar cycle 22
}

\author{
K.-J. Li ${ }^{1,2}$, B. Schmieder ${ }^{3,4}$, and Q.-Sh. $\mathbf{L i}^{2}$ \\ 1 CCAST (World Laboratory), P.O. Box 8730, Beijing, 100080, China \\ 2 Yunnan Observatory, Kunming, China \\ 3 Observatoire de Paris, Section de Meudon, URA 2080, 92195 Meudon Principal Cedex, France \\ ${ }^{4}$ University of Oslo, Blindern, P.O. Box 1029, Norway
}

Received March 19, 1997; accepted February 11, 1998

\begin{abstract}
The data of the X-ray flare events of class $M \geq 1$ during the interval of 1987 to 1992 corresponding to the maximum period of the 22nd solar cycle have been investigated to study the North-South $(\mathrm{N}-\mathrm{S})$ and WestEast (W-E) asymmetries. During that period it has been shown the existence of a real $\mathrm{N}-\mathrm{S}$ asymmetry. That confirms recent studies done by using other indicators, such as sunspot areas (Oliver \& Ballester 1994). The E-W asymmetry during that period is not significant but a non uniform flare distribution in longitude has been evidenced. That result is also in good agreement with the work made by Joshi (1995) using $\mathrm{H} \alpha$ flares for the same time period.

We have shown that the behaviour of the reverse tendency of Solar Cycle 21 to exhibit a predominance of the southern hemisphere in the $\mathrm{N}-\mathrm{S}$ asymmetry compared to the previous Solar Cycles (19 and 20) is maintained for Solar Cycle 22. A long period cycle of the N-S asymmetry could be suspected.
\end{abstract}

Key words: Sun: activity — Sun: flares — Sun: X-rays

\section{Introduction}

Solar activity indications do not occur evenly on the solar disk. It is well known that many types of solar phenomena exhibit some N-S asymmetry distributions (Reid 1968; Hansen \& Hansen 1975; Roy 1977; Swinson et al. 1986; Verma 1987; Verma et al. 1987; Garcia 1990; Oliver \& Ballester 1994; Atac \& Ozguc 1996; Joshi 1995; Heras et al. 1996). One of the first studies concerning the $\mathrm{N}-\mathrm{S}$ asymmetry was made by Newton \& Milson (1955), who studied the distribution of the yearly values of sunspot areas from 1874 to 1955 . Later on, different studies of N-S

Send offprint requests to: B. Schmieder asymmetry have been made based on different types of solar phenomena, relative sunspot numbers, sunspot areas, sunspot magnetic classes, sudden disappearances of solar filaments, type II radio bursts, white-light flares, gammaray bursts, hard X-ray bursts, events of coronal mass ejection, and X-ray flares (see the above references). These investigations have demonstrated the existence of a N-S asymmetry, its quasi periodic behavior of 11 years and its correlations with some phase difference with other physical phenomena. Although the existence of N-S asymmetry in solar activity is generally well established, it is not so well interpreted (Carbonell et al. 1993). Garcia (1990) studied the N-S asymmetry of large flares based on X-ray observations from GOES satellites during Solar Cycles 20 and 21 . He shows that the spatial distribution of flares varies with solar cycle such as that the preponderance of flares occurs in the North in the early part of the cycle and moves south as the cycle progresses. Bai (1987, 1988) remarked that active regions producing flares and active regions with low activity could originate from different levels of the convection zone. The first class of active regions could come from superactive zones with rotation periods substantially shorter than the Carrington rotation period and comparable to that of large scale magnetic polarity patterns (McIntosh 1981). McIntosh et al. (1985) proposed that this large scale pattern is the result of giant convective cells. The association of these observations leads Garcia to think that flares originate in active regions anchored deep in the convection zone, may be at the boundary of giant cells. So there is, may be, a relationship between the asymmetries and giant convective cells. A global convection pattern was recently discovered by analysing the data of MDI aboard SOHO (Kosovitchev et al. 1997) and large torsional zones have been confirmed. The existence of the torsional oscillations could be related to the rolls defined by Ribes et al. (1993) using young spots as tracers. They show that the rolls parallel to the 
equatorial plane describe well the meridian circulation. The number of such convective rolls changes according to the phase of the solar cycle from 3 at the onset of the cycle to 1 at the decay of the cycle. Because the sun is a non rigid body in rotation, the meridian circulation has as a consequence the differential rotation of the surface due to the transport of momentum and magnetic activity. All these works would give a new view on the solar dynamo phenomenon.

On the other hand, Verma (1987) who was working on 3 Solar Cycles 19, 20 and 21 with different indicators found that asymmetries in all phenomena prevailed in the north during cycles 19 and 20 and in the south in cycle 21 . This would indicate that superimposed on the well observed periodicities of 11, 22 years a much longer cycle of unknown duration does exist.

The existence of a $\mathrm{E}-\mathrm{W}$ asymmetry is more controversial. During short periods (1978-1980) heliolongitudinal distribution of intense flares shows an important asymmetry towards the east and it has been related to the large number of interplanetary shocks observed in the eastern hemisphere (Hewish \& Bravo 1986). Horas et al. (1990) found a pronounced asymmetry of flares and subflares between 1976-1985, although the asymmetry was much more important during periods close to the minimum of solar activity while around the maximum it was very small. Joshi (1995) using $\mathrm{H} \alpha$ flares found no $\mathrm{E}-\mathrm{W}$ asymmetry for Solar Cycle 21 and a small asymmetry during the Solar Cycle 22 . There is no obvious reason why the $\mathrm{W}-\mathrm{E}$ asymetry should exist over a long period. Some ideas have been proposed to explain why we could find a E-W asymmetry. Some gravitational effects with the interaction of Jupiter have been suggested in the past but it does not seem very promising (Kleczek private discussion). An other possibility is the influence over a few solar rotations of the transit of active flaring regions on the solar disk (Heras et al. 1990). In fact preferred heliolongitudes for solar activity have recently been pointed out by Bai $(1987,1988)$. Finally an other idea would be due to the pure geometry of the magnetic field in the sunspots. According to Bai (1988) and McIntosh (see above) the active regions are anchored deep in the convective zone. In these regions there are strong magnetic shears. The magnetic field lines when they emerge at the solar surface would have some inclination. So the area of sunspots could have different sizes in the western and in the eastern hemispheres according to the inclination of the field lines. If the angle is similar to this of the prominences, inclination of 15 degrees with the vertical towards the west (Tandberg-Hanssen 1974), the area of the sunspots should be larger on the eastern than on the western side. Again this is a topics of great importance with the recent development of seismic approachs with SOHO/MDI (Braun et al. 1993).

In this paper, we have used the data of the X-ray flares $(M \geq 1)$ during the maximum period of the 22 nd solar cycle in order to investigate the behavior of asymmetries:
$\mathrm{W}-\mathrm{E}$ asymmetry as well as $\mathrm{N}-\mathrm{S}$ asymmetry in order to complement the work done by Garcia (1990) for the previous solar cycles and to compare with the results of Joshi (1995) and Heras et al. (1990) concerning Solar Cycle 22 but using other indicators. We also study distributions of the flares with respect to the longitude. We confirm our results by using probability laws in order to check if they could be obtained quite by chance.

\section{Statistical analysis}

\subsection{X-ray flare data}

The data used in this paper come from the collection of the X-ray $M \geq 1$ flares (Li \& Zhang 1994), which collected all X-ray $M \geq 1$ flare events listed in SGD (Solar Geophysical Data) in the time interval of 1 April 1987 to 30 December 1992 corresponding to the maximum period of the 22nd solar cycle. The table gives the following parameters of each flare event: date, beginning time, maximum time, ending time, X-ray class, and position on the solar disk. The total number of the considered flare events is 2052 . The sample does not collect flare events before 1987 and after 1992, because their number was not large enough significant for a statistical analysis.

So we just use the data listed in their table and do not add any data to complete the analysis of an entire solar cycle. Table 1 gives the number of the flares classified by hemisphere and by X-ray class.

Here, S, N, E, and W stand for the southern, northern, eastern, and western hemispheres respectively; $M$ and $X$ stand for the X-ray flares of $M$ and $X$ classes (or importances) respectively; the last column gives the total number of the annual flare events, and the last row gives the total number in the flare-class within the considered six years.

\subsection{Distributions of the flare events on the solar disk}

\subsection{1. $\mathrm{N}-\mathrm{S}$ distribution}

The spatial distribution of flares in heliographic latitude is usually studied to determine whether there is a $\mathrm{N}-\mathrm{S}$ spatial asymmetry or not, and whether the asymmetry is dependent on the intensities of the flares, or any other associated characteristics. Figure 1 gives the annual number ratio of the flare events in the southern hemisphere $\left(N_{\mathrm{S}}=1226\right)$ to those in the northern hemisphere $\left(N_{\mathrm{N}}=\right.$ $826)$. There is no flare of $X$ class occurring in 1987, here we let the value $N_{\mathrm{S}} / N_{\mathrm{N}}$ of this year equal to 1 for the flares of $X$ class. The same is $N_{\mathrm{E}} / N_{\mathrm{W}}$ in Fig. 3. Figure 1 clearly shows that the flare events in the southern hemisphere are much more numerous than those in the northern hemisphere in five years of the six for $M$ class, $X$ class, and $M \& X$ class respectively. The ratio of the total number of 
Table 1. The classification number of $M \geq 1$ X-ray flares at the period of 1987 to 1992

\begin{tabular}{|c|c|c|c|c|c|c|c|c|c|}
\hline & $N_{o \cdot(S, M)}$ & $N_{\cdot}(S, X)$ & $N_{o \cdot(N, M)}$ & $N_{O}{ }_{(N, X)}$ & $N_{o}{ }_{(E, M)}$ & $\operatorname{No}_{(E, X)}$ & $N_{o}(W, M)$ & $N_{o}(W, X)$ & ${ }^{* *}$ total \\
\hline * 1987 & 18 & 0 & 11 & 0 & 12 & 0 & 17 & 0 & 29 \\
\hline 1988 & 111 & 10 & 81 & 3 & 112 & 3 & 80 & 10 & 205 \\
\hline 1989 & 347 & 32 & 274 & 27 & 325 & 29 & 296 & 30 & 680 \\
\hline 1990 & 121 & 2 & 151 & 14 & 142 & 11 & 130 & 5 & 288 \\
\hline 1991 & 398 & 37 & 186 & 17 & 298 & 36 & 286 & 18 & 638 \\
\hline 1992 & 142 & 8 & 58 & 4 & 80 & 1 & 120 & 11 & 212 \\
\hline total & 1137 & 89 & 761 & 65 & 969 & 80 & 929 & 74 & \\
\hline
\end{tabular}

* The flare events are summed up into the numbers of this row only from 1 April 1987.

** Number in this column corresponds to the number of the flare events of each year, the half of the sum $\sum$ No.

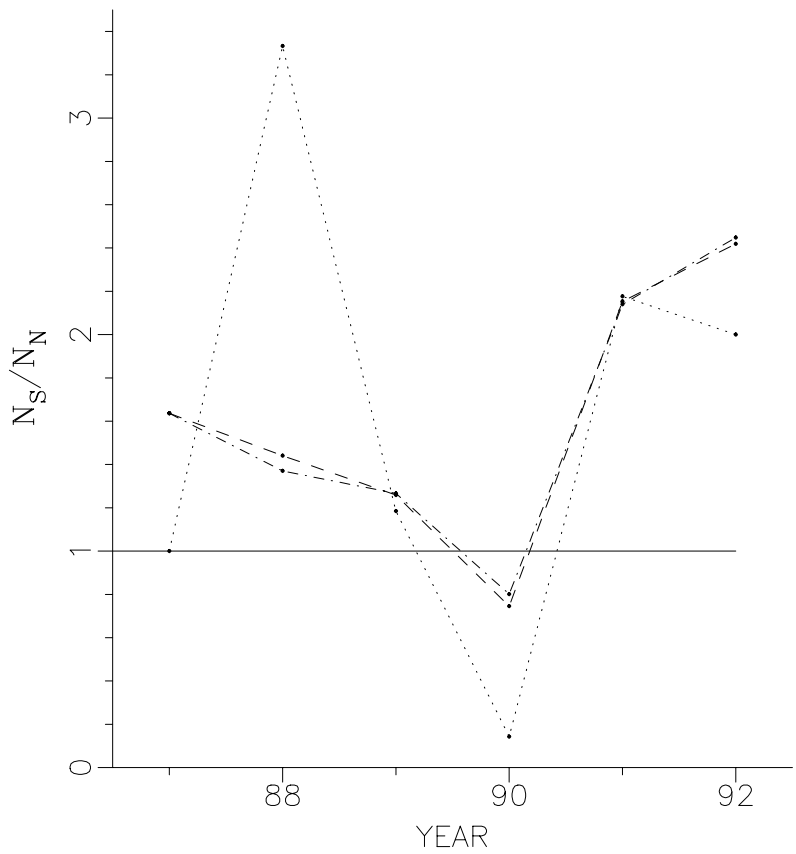

Fig. 1. The annual number ratio of the flare events in the southern hemisphere $\left(N_{\mathrm{S}}\right)$ to those in the northern hemisphere $\left(N_{\mathrm{N}}\right)$. All flare events of $M \& X$ classes are considered in the dashed curve, those of $M$ class in the dashed and dotted curve, and those of $X$ class in the dotted curve

the flare events in the southern hemisphere to that in the northern hemisphere is 1.49 for $M$ class, 1.37 for $X$ class, 1.48 for $M \& X$ class. An overall southern bias apparently prevailed in the solar cycle 22 . The above numbers indicate that the flare events of $M \& X$ class in the southern hemisphere exceeded the events in the northern hemisphere by an unexpected large percentage $48.6 \%$ during the investigated interval. This is a strong $\mathrm{N}-\mathrm{S}$ asymmetry.

To be sure that this result cannot be obtained purely by chance we check by using probability laws an compute what would be the ratio of flares in the southern hemisphere to those in the northern hemisphere that we can expect only by chance. Let us consider a distribution of $n$ objects (flares) in 2 classes. The probability that one flare (one object) occurs in one hemisphere (class one) by chance is $p=1 / 2$. We use the following binomial formula to derive the probability $P(k)$ of getting $k$ objects in class 1 and $n-k$ objects in class 2 .

$P(k)=\left(\begin{array}{c}n \\ k\end{array}\right) p^{k}(1-p)^{n-k}$.

In our case for a total number $n=N_{\mathrm{S}}+N_{\mathrm{N}}=2052$, we have for example:

$P(k)=\frac{2052 !}{(2052-k) ! k !} \frac{1}{2^{2052}}$.

The probability to get more than d objects in one class is:

$P(\geq d)=\sum_{k=d}^{n} P(k)$.

We have 1226 (class $M+X$ ) events occuring in the southern hemisphere, the probability to get such amount of events or even more by chance is $P(\geq 1226) \sim 10^{-8} .1137$ events occurred in the southern hemisphere within the total 1898 events of $M$ class, $P(\geq 1137) \sim 10^{-8} .89$ events within the total 154 events of $X$ class occurred in the southern hemisphere, $P(\geq 89)=0.026$. The probability to get by chance more than 1226 flares in the southern hemisphere is very weak, more than $1137 \mathrm{M}$ class flares is also weak, to get more than $89 \mathrm{X}$ events is less than $3 \%$. In general, when $P(\geq d)<5 \%$ we have a statistically significant result, and when $P(\geq d)<1 \%$ the result is highly significant. So the numbers $1.49,1.48$ and 1.37 are statistically significant. There really existed an N-S asymmetry for the flares of $M$ class, for the flare events of $X$ class, and for the total flare events of $M \& X$ class respectively.

These results are complimentary to several ones published recently. Oliver \& Ballester (1994) and Atac \& Ozguc (1996) stressed the dominance of southern hemisphere during most Solar Cycle 22 using sunspot areas and solar flare index, respectively. Such a result points out a possible trend in the behaviour of the $\mathrm{N}-\mathrm{S}$ asymmetry.

If globally it is in good agreement with previous studies, we did not find any variation with the flare class and any relation with the magnetic field flux obtained by some of these studies. Garcia (1990) found that the degree of $\mathrm{N}-\mathrm{S}$ asymmetry apparently is a function of the intensity 


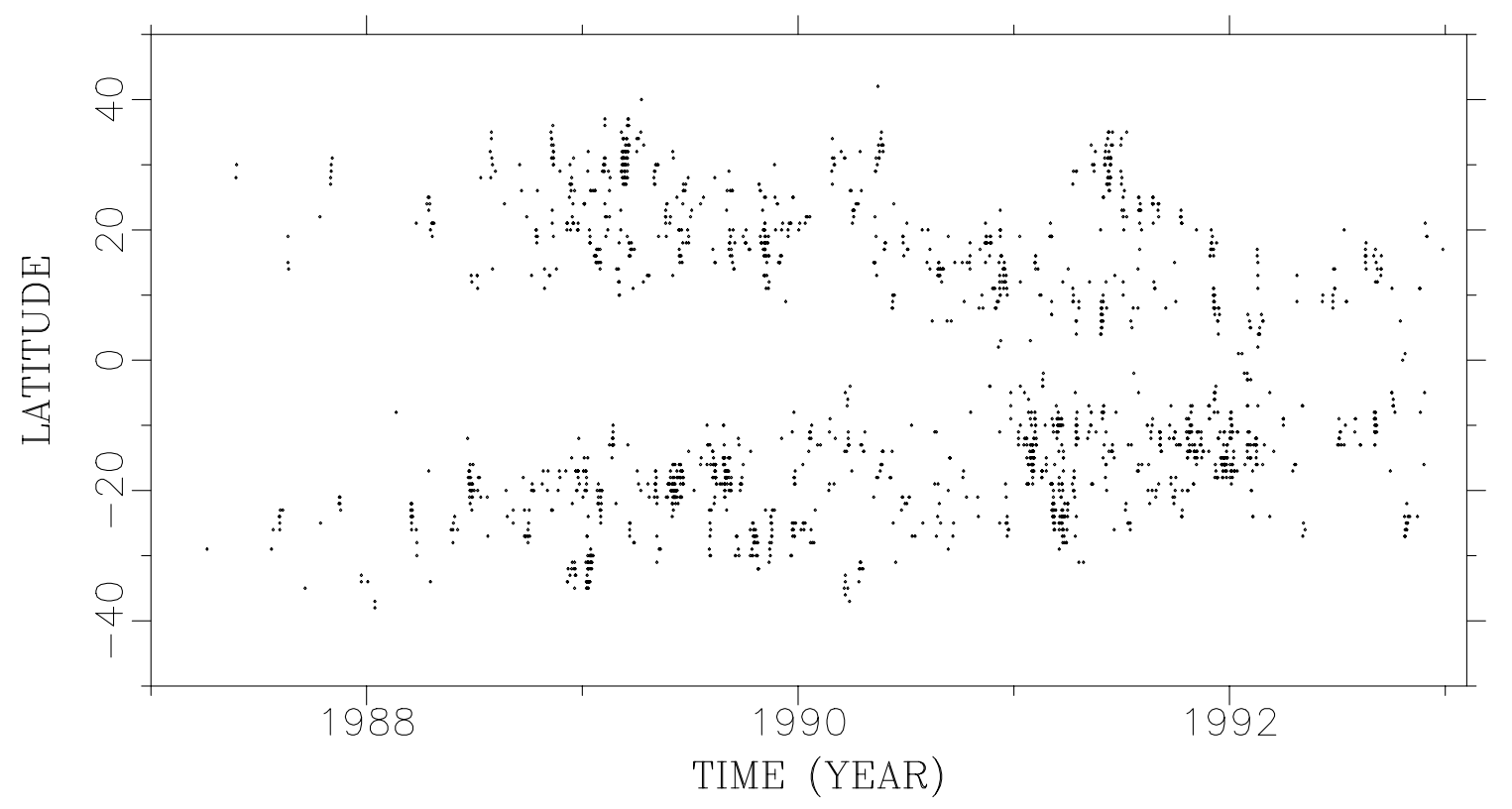

Fig. 2. Distribution of X-ray $M \geq 1$ flares from 1987 to 1992 , with respect to heliographic latitude

of the studied events, and the most intense events show the largest amount of $\mathrm{N}-\mathrm{S}$ asymmetry. This statement was valid for the solar Cycle 21 but according to our results it does not seem to be confirmed for Solar Cycle 22. Our statistical results show that the degree $(36.9 \%)$ of $\mathrm{N}-\mathrm{S}$ asymmetry for the flares of $X$ class is smaller than the degree $(49.4 \%)$ for the flares of $M$ class. Howard (1974) ever studied solar magnetic flux data for the period 1967 to 1973 . He found that the total flux in the northern hemisphere exceeded that in the southern hemisphere, but only by a small percentage $7 \%$. The discrepancy between our results and the Heras's results could be due to the fact that in the upper latitude the $\mathrm{N}-\mathrm{S}$ asymmetry flux is less pronounced than in the lower ones or more difficult to establish because of the weak statistics.

Further investigation of the relation between degree of $\mathrm{N}-\mathrm{S}$ asymmetry and intensity of studied events is needed in future. Heliographic latitude of each flare event is plotted in Fig. 2 with respect to the date of occurrence. Southern heliographic latitudes take the negative sign in the figure. Several features and peculiarities are immediately apparent. At the beginning of the 22nd solar cycle, the X-ray $M \geq 1$ flares occurred in the relatively high latitudes (above $20^{\circ}$ ), then, as the cycle is progressing, there was an obvious shift in the statistical centroïd position to the lower latitudes. The majority of the flare events occurred in the latitudes between $8^{\circ}$ and $35^{\circ}$. Only 2 flares occurred in the latitudes over $40^{\circ}$, and no flares occurred in the latitudes over $45^{\circ}$. Garcia (1990) gave the distribution of $M \geq 1$ flares from 1969 to 1984 with respect to the heliographic latitude in Figs. 2 and 3 of his paper and find similar results. The distribution in latitude of the flares is comparable to the sunspot distribution.

\subsection{2. $\mathrm{W}-\mathrm{E}$ distribution}

The annual number ratio of the flare events in the eastern hemisphere $\left(N_{\mathrm{E}}\right)$ to those in the western hemisphere $\left(N_{\mathrm{W}}\right)$ is plotted in Fig. 3. The flare events in the eastern hemisphere are more numerous than those in the western hemisphere in four years, which correspond to the maximum period. The ratio of the total number of flares in the eastern hemisphere to that in the western hemispheres is $969 / 929(=1.043)$ for $M$ class, $80 / 74(=1.083)$ for $X$ class, $1049 / 1003(=1.046)$ for $M \& X$ classses, and the corresponding actual probabilities $P(\geq d)$ are $0.179,0.314$, and 0.155 , respectively. In general, when $P(\geq d)>10 \%$ it implies a statistically insignificant result.

So the slight $\mathrm{W}-\mathrm{E}$ asymmetry that we have found in the distribution of the X-ray $M \geq 1$ flare events during the period of 1987 to 1992 cannot be considered significant in terms of $\mathrm{W}-\mathrm{E}$ asymmetry. Heliographic longitude of each flare event is plotted in Fig. 4 with respect to the date of occurrence. Western heliographic longitudes take the negative sign in the figure. The events are spread in all the longitudes.

The $\chi^{2}$ test can be used to check the null-hypothesis whether the longitude distribution of the events is due quite by chance. In order to check this point we divide firstly the events into nine longitude ranges, and the number $f_{i}$ of the events in each range is listed in Table 2 . Then we calculate the value of $\chi^{2}$ :

$\chi^{2}=\sum_{i=1}^{n} \frac{\left(f_{i}-\bar{f}\right)^{2}}{\bar{f}}$

where $\bar{f}=\frac{\sum_{i=1}^{n} f_{i}}{n}$ and $\mathrm{n}=9$. According to Eq. (3) and Table 2 we have $\chi^{2}=33.27$. Looking at the tables of critical $\chi^{2}$ with $n-1$ degree of freedom corresponding to 
Table 2. Number of the flare events in different longitude ranges

\begin{tabular}{|c|c|c|c|c|c|c|c|c|c|}
\hline Longitude range & {$[90,70]$} & $\overline{(70,50]}$ & $\overline{(50,30]}$ & $(30,10]$ & $(10,-10]$ & $(-10,-30]$ & $(-30,-50]$ & $(-50,-70]$ & $(-70,-90]$ \\
\hline Number & 168 & 265 & 262 & 250 & 222 & 222 & 243 & 214 & 206 \\
\hline
\end{tabular}

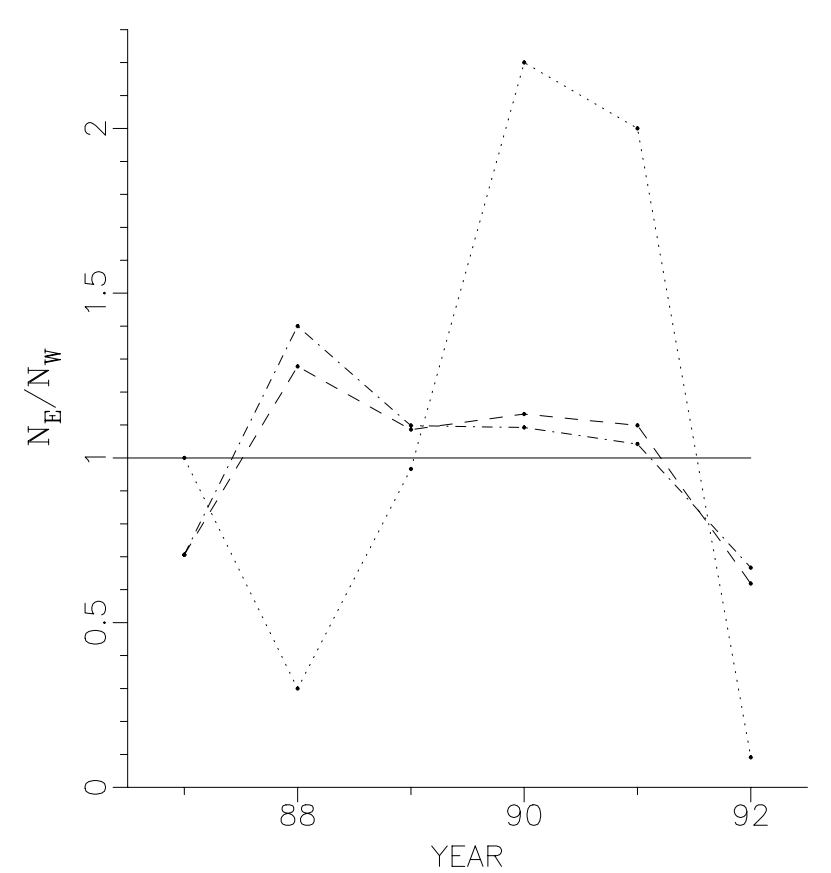

Fig. 3. The annual number ratio of the flare events in the eastern hemisphere $\left(N_{\mathrm{E}}\right)$ to those in the western hemisphere $\left(N_{\mathrm{W}}\right)$. All flare events of $M$ and $X$ classes are considered in the dashed curve, those of $M$ class considered in the dashed and dotted curve, and those of $X$ class considered in the dotted curve

our null hypothesis we find for example that the critical value of $\chi^{2}$ distribution $\chi_{0.005}^{2}(8)=21.9$, which is less than our value $\chi^{2}$. It means that the probability to have the observed distribution by chance is less than $5 / 1000$. So, we can consider that the events are not uniformly distributed in the longitude.

\section{Discussions and conclusions}

In this paper, we use the data of the $\mathrm{X}$-ray flare $(M \geq 1)$ events during the interval of 1987 to 1992 corresponding to the maximum period of the $22 \mathrm{nd}$ solar cycle in order to investigate the behavior of $\mathrm{N}-\mathrm{S}$ and $\mathrm{W}-\mathrm{E}$ asymmetries. In the present study we have found that the N-S asymmetry really existed for the flare events with the dominance of the southern hemisphere. The solar cycle 22 seems to have the same behaviour than the cycle 21 in respect to the $\mathrm{N}-\mathrm{S}$ asymmetry. This study is complementary to the work of Garcia (1990) concerning his analysis using X-ray flares for Solar Cycles 20 and 21 and is in good agreement with studies of Solar Cycle 22 using different indicators (Verma 1992; Oliver \& Ballester 1994; Joshi 1995).

The distribution of the X-ray $M \geq 1$ flares from 1987 to 1992 with respect to heliographic longitude shows that the flares were not uniformly distributed in longitude but we did not find any significant $\mathrm{W}-\mathrm{E}$ asymmetry during the period, which confirms the results of Joshi (1995) for the same period of time.

The study of $\mathrm{N}-\mathrm{S}$ asymmetry has to be continued in order to find some relationship between the existence of torsional oscillations observed by MDI on SOHO and the convection rolls suspected by Ribes et al. (1993) to explain the meridional circulation. All these studies would allow us to have a better insight into the solar dynamo system.

On the other hand, a long term study of N-S asymmetry has to be implemented to prove the existence of a long duration solar cycle superimposed to the 11 Solar Cycle which could correspond to the existence of a global magnetic field of long period variability (Verma 1987).

Acknowledgements. The author (LkJ) gratefully acknowledges the useful help in the input of the data used in this paper into a VAX computer from Zhang Xiaoyu of Yunnan Observatory and the help from P. Micheneau during his stay in DASOP, Meudon Observatory.

The author (LkJ) would like to express his gratitude for support and kind hospitality at the Observatoire during his scholar visit. The author (LKJ) is also indebted to Mr. Zhong Shuhua for his help and support.

We thank particularly Drs. Kleczek and Ballester who showed their interest in this study and favoured fruitful discussions which are partially reproduced in this paper, Dr G.Chambe who checked the probability computations and the unknown referee who helped us to clarify the manuscript.

\section{References}

Atac T., 1987, A\&A 135, 201

Atac T., Ozguc A., 1996, Solar Phys. 166, 201

Bai T., 1987, ApJ 314, 795

Bai T., 1988, ApJ 328, 860

Braun D.C., Duvall T.L., LaBonte B.J., Jefferies S.M., Pomerantz M.A., Harvey J.W., 1993, "Seismic Investigation of the Sun and Stars". Astronomical Society of the Pacific Publ., p. 77

Garcia H.A., 1990, Solar Phys. 127, 185

Carbonell M., Oliver R., Ballester J.C., 1993, A\&A 274, 497

Hansen R., Hansen S., 1985, Solar Phys. 44, 225

Heras A.M., Sanahuja B., Shea M.A., Smart D.F., 1990, Solar Phys. 126, 371

Hewish A., Bravo S., 1986, Nat 324, 601 


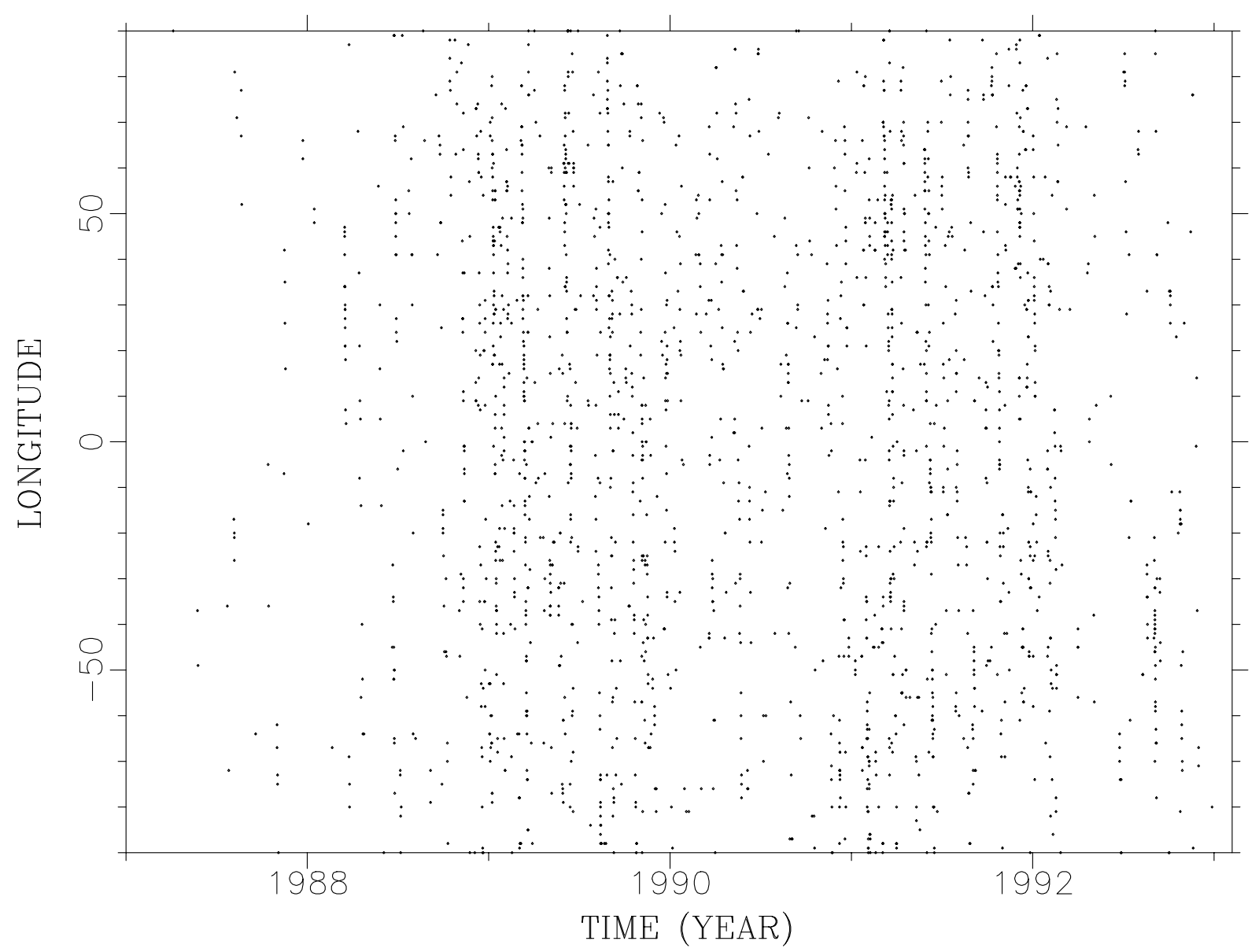

Fig. 4. Distribution of X-ray $M \geq 1$ flares from 1987 to 1992, with respect to heliographic longitude

Howard R., 1974, Solar Phys. 38, 59

Joshi A., 1995, Solar Phys. 157, 315

Kosovichev A.G., 1997, IAU Colloquium in Kyoto, August 1997

Li Qiusha, Zhang Bairong, 1994, Publ. Yunnan Obs. 54, 1

Newton H.W., Milson A.S., 1955, MNRAS 115, 398

McIntosh P.S, 1981, in The plasma of Sunspots, Sac Peak, L.E. Cram and Thomas J.H. (eds.)

McIntosh P.S, Wilson P.R., 1985, Solar Phys. 97, 59

Oliver R., Ballester J.L., 1994, Solar Phys. 152, 481
Reid J.H., 1968, Solar Phys. 5, 207

Ribes E., Ferreira E.N., Mein P., 1993, A\&A 274, 563

Roy J.R., 1977, Solar Phys. 52, 53

Tandberg-Hanssen E., 1974, "Solar Prominences". D. Reidel Publ. Co: Dordrecht, Holland

Verma V.K., 1987, Solar Phys. 114, 185

Verma V.K., Pande M.C., Uddin W., 1987, Solar Phys. 112, 341

Verma V.K., 1992, The Solar Cycle, ASP Conf. Ser. 27, 429 